\title{
CEPHEID RADII FROM INFRARED PHOTOMETRY
}

\author{
D.L. Welch \\ Dept. of. Astronomy, University of Toronto \\ Nancy Remage Evans \\ Dept. of Astronomy, University of Toronto \\ G. Drukier \\ Dept. of Astronomy, University of Toronto
}

\begin{abstract}
We have determined radii for Cepheids using nearinfrared $\mathrm{K}(2.2 \mu \mathrm{m})$ photometry and Balona's approach to the Baade-Wesselink method of radius determination. The lower sensitivity of the $K$ flux to temperature variations results in improved sensitivity to radius changes. We present the results of maximum-likelihood radius solutions for Cepheids with modern radial velocity curves. We point out limitations of the Balona technique as it is currently applied in the optical and suggest improvements.
\end{abstract}

\section{Introduction}

The goal of Baade-Wesselink techniques of radius determination is to remove the effects of temperature from the total flux. The remaining flux variation can then be interpreted in terms of radius changes. It makes sense to choose a photometric bandpass where temperature variations affect the flux as little as possible. The flux at $\mathrm{V}$ is strongly influenced by temperature as witnessed by the strong resemblance of the $V$ and $B-V$ curves for Cepheids. We suggest that nearinfrared bandpasses, such as $K$, are to be preferred for radius determination.

\section{Procedure}

We adopt here the technique of Balona (1977). This method results in a single equation of condition for light, color, and radius displacement. The equations are solved using the method of maximum likelihood as all quantities contain uncertainties. The Balona method assumes that both $\log \mathrm{T}_{e}$ and the bolometric correction are linearly dependent on $(B-V)_{0}$. The method is discussed in more detail in a paper presently in preparation.

Long recognized as a major complication of radius determination is the problem of phase-mismatch between the light/color curves and the radial velocity curve. We used radial velocity curves for which the uncertainty in the phase match is $0.01 \mathrm{p}$ or less. Photometry is from Welch et al. (1984); radial velocity curves have been taken from a number of sources; color curves have been taken from Gieren (1981) and Moffett and Barnes (1980). We avoided Cepheids with bright blue companions. Radii determined from $K$ photometry and optical (B-V) photometry are shown in Table 1 , together with surface brightness coefficients, $S_{K}$. One sigma errors are given. 
Table 1

$K$ Surface Brightness Coefficients and Radii for Cepheids

\begin{tabular}{lccrrr} 
& $\log P$ & \multicolumn{2}{c}{$\mathrm{S}_{\mathrm{K}}$} & \multicolumn{2}{c}{$\mathrm{R} / \mathrm{R}_{\odot}$} \\
SS Sct & 0.565 & 0.26 & \pm 0.06 & 37.3 & \pm 4.6 \\
T Vul & 0.647 & 0.42 & 0.03 & 39.1 & 1.6 \\
BB Sgr & 0.822 & 0.56 & 0.03 & 42.1 & 1.8 \\
U Sgr & 0.829 & 0.40 & 0.06 & 51.6 & 4.3 \\
V496 Aq1 & 0.833 & 0.73 & 0.08 & 41.5 & 3.7 \\
S Sge & 0.923 & 0.30 & 0.02 & 67.1 & 2.9 \\
X Cyg & 1.215 & 0.65 & 0.02 & 85.7 & 2.5 \\
Y Oph & 1.234 & 0.40 & 0.04 & 105.9 & 7.1
\end{tabular}

Discussion

A problem with the interpretation of the slope of the surface brightness-color relation (A in Balona's formulation) arises because of the wavelength dependence of reddening. If $E(B-V)$ is taken to be constant, the ratio of total-to-selective absorption (R) is a function of $(B-V)$. Olson (1975) and Grieve (1983) find values for $\mathrm{dP} / \mathrm{d}(\mathrm{B}-\mathrm{V})_{0}$ of 0.25 and 0.50 , respectively. Therefore, Balona's $A$ will be the sum of the true slope of the surface brightness-color relation and $\left[\mathrm{dR} / \mathrm{d}(\mathrm{B}-\mathrm{V})_{0}\right] E(B-V)$. This means that the true slope $A$ is typically smaller than Balona's average $(2.15)$ by 0.05 to 0.15 . This results in a small but systematic shift in the absolute magnitude calibration of Martin, Warren, and Feast (1979) in the sense that the derived magnitudes are brighter. It is possible to remove the wavelength dependence of the reddening by assuming a functional form for $\left.R(B-V)_{0}\right)$, choosing $E(B-V)$ and then iterating to $(B-V)_{0}$. Balona has previously interpreted high values of $A$ as evidence for a companion. The above discussion suggests that reddening may often be the cause.

\section{References}

Balona, L.A. 1977, M.N.R.A.S., 178, 231.

Gieren, W. 1981, Ap. J. Suppl., $\overline{47}, 315$.

Grieve, G.R. 1983, Ph.D. Thesis, Univ, of Toronto.

Martin, W.L., Warren, P.R., and Feast, M.W. 1979, M.N.R.A.S., $188,139$.

Moffett, T.J. and Barnes, T.G. 1980, Ap. J. Supp1., 44, 427. 01son, B.I. 1975, P.A.S.P., 87, 349.

Welch, D.L., Wieland, F., McĀary, C.W., McGonegal, R., Madore, B.F., McLaren, R.A., and Neugebauer, G. 1984 Ap. J. Supp1., 54, 547. 